\title{
On the D-wave state component of the deuteron in the Nambu-Jona-Lasinio model of light nuclei
}

\author{
A. N. Ivanov $* \ddagger$, V. A. Ivanova ${ }^{\ddagger}$, H. Oberhummer ${ }^{\dagger}$, \\ N. I. Troitskaya ${ }^{\ddagger}$, M. Faber $\$$
}

October 29, 2018

\begin{abstract}
Atominstitut der Österreichischen Universitäten, Arbeitsbereich Kernphysik und Nukleare Astrophysik, Technische Universität Wien, Wiedner Hauptstr. 8-10, A-1040 Wien, Österreich
\end{abstract}

\begin{abstract}
The D-wave state component of the neutron-proton bound state in the deuteron is calculated in the Nambu-Jona-Lasinio model of light nuclei. For the ratio of Dto S-state deuteron wave functions we obtain equal to $\eta_{\mathrm{d}}=0.0238$. This agrees well with the phenomenological value $\eta_{\mathrm{d}}=0.0256 \pm 0.0004$ quoted by Kamionkowski and Bahcall (ApJ. 420, 884 (1994)).
\end{abstract}

PACS: 11.10.Ef, 13.75.Cs, 14.20.Dh, 21.30.Fe

Keywords: field theory, QCD, deuteron, neutron, proton, nucleon- nucleon scattering

\footnotetext{
${ }^{*}$ E-mail: ivanov@kph.tuwien.ac.at, Tel.: +43-1-58801-14261, Fax: +43-1-58801-14299

${ }^{\dagger} \mathrm{E}-$ mail: ohu@kph.tuwien.ac.at, Tel.: +43-1-58801-14251, Fax: +43-1-58801-14299

${ }^{\ddagger}$ Permanent Address: State Technical University, Department of Nuclear Physics, 195251 St. Petersburg, Russian Federation

${ }^{\S}$ E-mail: faber@kph.tuwien.ac.at, Tel.: +43-1-58801-14261, Fax: +43-1-58801-14299

IThe authors are ordered according to Russian alphabet as usual for publications $[1,2,3]$
} 


\section{Introduction}

The Nambu-Jona-Lasinio model of light nuclei or differently the nuclear NambuJona-Lasinio (NNJL) model suggested in [1-3] represents a relativistically covariant quantum field theoretic approach to the description of low-energy properties and interactions of the deuteron and light nuclei. The NNJL model is fully motivated by QCD [1]. The deuteron appears in the nuclear phase of $Q C D$ as a neutron-proton collective excitation, the Cooper np-pair, induced by a phenomenological local four-nucleon interaction. The NNJL model describes low-energy nuclear forces in terms of one-nucleon loop exchanges providing a minimal transfer of nucleon flavours from initial to final nuclear states and accounting for contributions of nucleon-loop anomalies which are completely determined by one-nucleon loop diagrams. The dominance of contributions of nucleon-loop anomalies to effective Lagrangians of low-energy nuclear interactions is justified in the large $N_{C}$ expansion, where $N_{C}$ is the number of quark colours.

Nowadays there is a consensus concerning the existence of non-nucleonic degrees of freedom in nuclei [4]. The non-nucleonic degrees of freedom can be described either within QCD in terms of quarks and gluons [5] or in terms of mesons and nucleon resonances [6]. In the NNJL model the non-nucleonic degrees of freedom of nuclei have been investigated in terms of the $\Delta(1232)$ resonance and calculated the contribution of the $\Delta \Delta$ component to the deuteron [2]. The obtained result $P(\Delta \Delta)=0.3 \%$ agrees well with the experimental upper bound $P(\Delta \Delta)<0.4 \%$ [7] and other theoretical estimates [4].

As has been shown in [3] the NNJL model describes well low-energy nuclear forces for electromagnetic and weak nuclear reactions with the deuteron of astrophysical interest such as the neutron-proton radiative capture $\mathrm{n}+\mathrm{p} \rightarrow \mathrm{D}+\gamma$, the solar proton burning $\mathrm{p}+\mathrm{p} \rightarrow \mathrm{D}+\mathrm{e}^{+}+\nu_{\mathrm{e}}$, the pep-process $\mathrm{p}+\mathrm{e}^{-}+\mathrm{p} \rightarrow \mathrm{D}+\nu_{\mathrm{e}}$ and reactions of the disintegration of the deuteron by neutrinos and anti-neutrinos caused by charged $\nu_{\mathrm{e}}+\mathrm{D}$ $\rightarrow \mathrm{e}^{-}+\mathrm{p}+\mathrm{p}, \bar{\nu}_{\mathrm{e}}+\mathrm{D} \rightarrow \mathrm{e}^{+}+\mathrm{n}+\mathrm{n}$ and neutral $\nu_{\mathrm{e}}\left(\bar{\nu}_{\mathrm{e}}\right)+\mathrm{D} \rightarrow \nu_{\mathrm{e}}\left(\bar{\nu}_{\mathrm{e}}\right)+\mathrm{n}+\mathrm{p}$ weak currents.

The important problem which has not been jet clarified in the NNJL model is related to the value of the contribution of the $D$-wave state to the wave function of the deuteron. In this paper we fill this blank. In section 2 we calculate the contribution of the Dwave state to the wave function of the deuteron. We use a relativistically covariant partial-wave analysis developed by Anisovich et al. [8] for the description of nucleonnucleon scattering. The fraction of the D-wave state of the deuteron wave function relative to the $\mathrm{S}-$ wave one we obtain equal to $\eta_{d}=0.0238$. This agrees well with the value $\eta_{d}=0.0256 \pm 0.0004$ quoted by Kamionkowski and Bahcall [9] $]$ who used this parameter for the phenomenological description of the realistic wave function of the deuteron in connection with the calculation of the astrophysical factor $S_{\mathrm{pp}}(0)$ for the solar proton burning $\mathrm{p}+\mathrm{p} \rightarrow \mathrm{D}+\mathrm{e}^{+}+\nu_{\mathrm{e}}$ in the potential model approach. In the Conclusion we discuss the obtained result.

\footnotetext{
${ }^{1}$ The value $\eta_{d}=0.0256 \pm 0.0004$ was taken by Kamionkowski and Bahcall from Ref. [10].
} 


\section{The D-wave state component of the deuteron}

The calculation of the value of the $\mathrm{D}$-wave state contribution to the wave function of the deuteron we would carry out in terms of the amplitude of the transition $\mathrm{n}+\mathrm{p} \rightarrow \mathrm{D}$. We show that the neutron-proton pair couples to the deuteron in both the S-wave state and the $\mathrm{D}$-wave state with the fraction of the $\mathrm{D}$-wave state agreeing with low-energy nuclear phenomenology.

In the NNJL model the phenomenological Lagrangian of the $\mathrm{npD}$ interaction is defined by $[1]$

$$
\begin{aligned}
\mathcal{L}_{\mathrm{npD}}(x)= & -i g_{\mathrm{V}}\left[\bar{p}^{c}(x) \gamma^{\mu} n(x)-\bar{n}^{c}(x) \gamma^{\mu} p(x)\right] D_{\mu}^{\dagger}(x) \\
& +\frac{g_{\mathrm{T}}}{2 M_{\mathrm{N}}}\left[\bar{p}^{c}(x) \sigma^{\mu \nu} n(x)-\bar{n}^{c}(x) \sigma^{\mu \nu} p(x)\right] D_{\mu \nu}^{\dagger}(x)+\text { h.c. }
\end{aligned}
$$

where $D_{\mu}^{\dagger}(x), n(x)$ and $p(x)$ are the interpolating fields of the deuteron, the neutron and the proton, $D_{\mu \nu}^{\dagger}(x)=\partial_{\mu} D_{\nu}^{\dagger}(x)-\partial_{\nu} D_{\mu}^{\dagger}(x)$ is the deuteron field strength. The phenomenological coupling constant $g_{\mathrm{V}}$ is related to the electric quadrupole moment of the deuteron $Q_{\mathrm{D}}=0.286 \mathrm{fm}^{2}, g_{\mathrm{V}}^{2}=2 \pi^{2} Q_{\mathrm{D}} M_{\mathrm{N}}^{2}[1]$, where $M_{\mathrm{N}}=940 \mathrm{MeV}$ is the nucleon mass. The coupling constants $g_{\mathrm{V}}$ and $g_{\mathrm{T}}$ are connected by the relation [1]

$$
g_{\mathrm{T}}=\sqrt{\frac{3}{8}} g_{\mathrm{V}}
$$

which is valid at leading order in the large $N_{C}$ expansion [1].

The amplitude of the transition $\mathrm{n}+\mathrm{p} \rightarrow \mathrm{D}$ is determined by

$$
\left\langle k_{\mathrm{D}}, \lambda_{\mathrm{D}}\left|\mathcal{L}_{\mathrm{npD}}(0)\right| k_{\mathrm{p}}, \sigma_{\mathrm{p}} ; k_{\mathrm{n}}, \sigma_{\mathrm{n}}\right\rangle=\frac{M\left(\mathrm{n}\left(\mathrm{k}_{\mathrm{n}}, \sigma_{\mathrm{n}}\right)+\mathrm{p}\left(\mathrm{k}_{\mathrm{p}}, \sigma_{\mathrm{p}}\right) \rightarrow \mathrm{D}\left(k_{\mathrm{D}}, \lambda_{\mathrm{D}}\right)\right)}{\sqrt{2 E_{\mathrm{D}} V 2 E_{\mathrm{n}} V 2 E_{\mathrm{p}} V}},
$$

where $\left(E_{\mathrm{D}}, k_{\mathrm{D}}, \lambda_{\mathrm{D}}\right),\left(E_{\mathrm{p}}, k_{\mathrm{p}}, \sigma_{\mathrm{p}}\right)$ and $\left(E_{\mathrm{n}}, k_{\mathrm{n}}, \sigma_{\mathrm{n}}\right)$ are energies, 4-momenta and polarizations of the deuteron, the proton and the neutron, respectively, $V$ is a normalization space volume. The wave functions of the initial and the final states of the transition $n+$ $\mathrm{p} \rightarrow \mathrm{D}$ are given by

$$
\begin{aligned}
\left|k_{\mathrm{p}}, \sigma_{\mathrm{p}} ; k_{\mathrm{n}}, \sigma_{\mathrm{n}}\right\rangle & =a_{\mathrm{p}}^{\dagger}\left(k_{\mathrm{p}}, \sigma_{\mathrm{p}}\right) a_{\mathrm{n}}^{\dagger}\left(k_{\mathrm{n}}, \sigma_{\mathrm{n}}\right)|0\rangle, \\
\left\langle k_{\mathrm{D}}, \lambda_{\mathrm{D}}\right| & =\langle 0| a_{\mathrm{D}}\left(k_{\mathrm{D}}, \lambda_{\mathrm{D}}\right),
\end{aligned}
$$

where $a_{\mathrm{p}}^{\dagger}\left(k_{\mathrm{p}}, \sigma_{\mathrm{p}}\right)$ and $a_{\mathrm{n}}^{\dagger}\left(k_{\mathrm{n}}, \sigma_{\mathrm{n}}\right)$ are creation operators of the proton and the neutron, $a_{\mathrm{D}}\left(k_{\mathrm{D}}, \lambda_{\mathrm{D}}\right)$ is the annihilation operator of the deuteron and $|0\rangle$ is a vacuum wave function. The relativistically invariant amplitude $M\left(\mathrm{n}\left(\mathrm{k}_{\mathrm{n}}, \sigma_{\mathrm{n}}\right)+\mathrm{p}\left(\mathrm{k}_{\mathrm{p}}, \sigma_{\mathrm{p}}\right) \rightarrow \mathrm{D}\left(k_{\mathrm{D}}, \lambda_{\mathrm{D}}\right)\right)$ reads

$$
\begin{aligned}
& M\left(\mathrm{n}\left(\mathrm{k}_{\mathrm{n}}, \sigma_{\mathrm{n}}\right)+\mathrm{p}\left(\mathrm{k}_{\mathrm{p}}, \sigma_{\mathrm{p}}\right) \rightarrow \mathrm{D}\left(k_{\mathrm{D}}, \lambda_{\mathrm{D}}\right)\right)=e^{* \nu}\left(k_{\mathrm{D}}, \lambda_{\mathrm{D}}\right) \\
& \times\left\{2 i g_{\mathrm{V}}\left[\bar{u}^{c}\left(k_{\mathrm{n}}, \sigma_{\mathrm{n}}\right) \gamma_{\nu} u\left(k_{\mathrm{p}}, \sigma_{\mathrm{p}}\right)\right]-\frac{2 i g_{\mathrm{T}}}{M_{\mathrm{N}}}\left[\bar{u}^{c}\left(k_{\mathrm{n}}, \sigma_{\mathrm{n}}\right) \sigma_{\mu \nu} u\left(k_{\mathrm{p}}, \sigma_{\mathrm{p}}\right)\right]\left(k_{\mathrm{n}}+k_{\mathrm{p}}\right)^{\mu}\right\},
\end{aligned}
$$

where $\bar{u}^{c}\left(k_{\mathrm{n}}, \sigma_{\mathrm{n}}\right)$ and $u\left(k_{\mathrm{p}}, \sigma_{\mathrm{p}}\right)$ are bispinorial wave functions of the neutron and the proton with 4-momenta $k_{\mathrm{n}}, k_{\mathrm{p}}$ and polarizations $\sigma_{\mathrm{n}}, \sigma_{\mathrm{p}} ; e^{* \nu}\left(k_{\mathrm{D}}, \lambda_{\mathrm{D}}\right)$ is a 4-vector of polarization 
of the deuteron with a $4-$ momentum $k_{\mathrm{D}}$ and polarization $\lambda_{\mathrm{D}}$. The 4 -momenta $k_{\mathrm{D}}, k_{\mathrm{n}}$ and $k_{\mathrm{p}}$ are related by $k_{\mathrm{D}}=k_{\mathrm{n}}+k_{\mathrm{p}}$ due to conservation of energy and momentum.

As has been shown by Anisovich et. al. [8] for neutron-proton scattering the neutronproton densities describing the $\mathrm{S}$ - and $\mathrm{D}$-wave states of a neutron-proton pair are equal to

$$
\begin{aligned}
\Psi_{\nu}\left({ }^{3} \mathrm{~S}_{1} ; \sigma_{\mathrm{n}}, \sigma_{\mathrm{p}}\right) & =\left[\bar{u}^{c}\left(k_{\mathrm{n}}, \sigma_{\mathrm{n}}\right) \mathcal{S}_{\nu} u\left(k_{\mathrm{p}}, \sigma_{\mathrm{p}}\right)\right], \\
\Psi_{\nu}\left({ }^{3} \mathrm{D}_{1} ; \sigma_{\mathrm{n}}, \sigma_{\mathrm{p}}\right) & =\left[\bar{u}^{c}\left(k_{\mathrm{n}}, \sigma_{\mathrm{n}}\right) \mathcal{D}_{\nu} u\left(k_{\mathrm{p}}, \sigma_{\mathrm{p}}\right)\right],
\end{aligned}
$$

where $\mathcal{S}_{\nu}$ and $\mathcal{D}_{\nu}$ are relativistically covariant operators of the projection onto the S-wave and the D-wave state, respectively [8]:

$$
\begin{aligned}
& \mathcal{S}_{\nu}=\frac{1}{\sqrt{2 s}}\left[\gamma_{\nu}^{\perp}-\frac{2 k_{\nu}}{2 M_{\mathrm{N}}+\sqrt{s}}\right], \\
& \mathcal{D}_{\nu}=\frac{2}{s^{3 / 2}}\left[\frac{1}{4}\left(4 M_{\mathrm{N}}^{2}-s\right) \gamma_{\nu}^{\perp}-\left(M_{\mathrm{N}}+\sqrt{s}\right) k_{\nu}\right] .
\end{aligned}
$$

Here $P=k_{\mathrm{p}}+k_{\mathrm{n}}, k=\frac{1}{2}\left(k_{\mathrm{p}}-k_{\mathrm{n}}\right), s=P^{2}, P \cdot k=0$ and

$$
\gamma_{\nu}^{\perp}=\gamma_{\nu}-\hat{P} \frac{P_{\nu}}{s}
$$

The neutron-proton densities Eq.(2.6) are normalized by the condition [8]

$$
\frac{1}{3} \int \operatorname{tr}\left\{L_{\mu}\left(\hat{k}_{\mathrm{p}}+M_{\mathrm{N}}\right) L^{\mu}\left(-\hat{k}_{\mathrm{n}}+M_{\mathrm{N}}\right)\right\}(2 \pi)^{4} \delta^{(4)}\left(P-k_{\mathrm{p}}-k_{\mathrm{n}}\right) \frac{d^{3} k_{\mathrm{p}}}{(2 \pi)^{3} 2 E_{\mathrm{p}}} \frac{d^{3} k_{\mathrm{n}}}{(2 \pi)^{3} 2 E_{\mathrm{n}}}=\rho_{L}(s),
$$

where $L_{\mu}=\mathcal{S}_{\mu}$ or $\mathcal{D}_{\mu}$, the factor 3 in the denominator of the 1.h.s. describes the number of the states of a neutron-proton density with a total momentum $J=1,2 J+1=3$, and $\rho_{\mathcal{S}}(s)$ and $\rho_{\mathcal{D}}(s)$ amount to

$$
\begin{aligned}
\rho_{\mathcal{S}}(s) & =\frac{1}{8 \pi}\left(\frac{s-4 M_{\mathrm{N}}^{2}}{s}\right)^{1 / 2}, \\
\rho_{\mathcal{D}}(s) & =\frac{1}{8 \pi}\left(\frac{s-4 M_{\mathrm{N}}^{2}}{s}\right)^{5 / 2} .
\end{aligned}
$$

In the center of mass frame of the neutron-proton pair the densities Eq.(2.6) are equal to

$$
\begin{aligned}
\Psi_{0}\left({ }^{3} \mathrm{~S}_{1} ; \sigma_{\mathrm{n}}, \sigma_{\mathrm{p}}\right) & =\left[\bar{u}^{c}\left(k_{\mathrm{n}}, \sigma_{\mathrm{n}}\right) \mathcal{S}_{0} u\left(k_{\mathrm{p}}, \sigma_{\mathrm{p}}\right)\right]=0 \\
\vec{\Psi}\left({ }^{3} \mathrm{~S}_{1} ; \sigma_{\mathrm{n}}, \sigma_{\mathrm{p}}\right) & =\left[\bar{u}^{c}\left(k_{\mathrm{n}}, \sigma_{\mathrm{n}}\right) \overrightarrow{\mathcal{S}} u\left(k_{\mathrm{p}}, \sigma_{\mathrm{p}}\right)\right]=\frac{1}{\sqrt{2}} \varphi_{\mathrm{n}}^{\dagger}\left(\sigma_{\mathrm{n}}\right) \vec{\sigma} \varphi_{\mathrm{p}}\left(\sigma_{\mathrm{p}}\right), \\
\Psi_{0}\left({ }^{3} \mathrm{D}_{1} ; \sigma_{\mathrm{n}}, \sigma_{\mathrm{p}}\right) & =\left[\bar{u}^{c}\left(k_{\mathrm{n}}, \sigma_{\mathrm{n}}\right) \mathcal{D}_{0} u\left(k_{\mathrm{p}}, \sigma_{\mathrm{p}}\right)\right]=0 \\
\vec{\Psi}\left({ }^{3} \mathrm{D}_{1} ; \sigma_{\mathrm{n}}, \sigma_{\mathrm{p}}\right) & =\left[\bar{u}^{c}\left(k_{\mathrm{n}}, \sigma_{\mathrm{n}}\right) \overrightarrow{\mathcal{D}} u\left(k_{\mathrm{p}}, \sigma_{\mathrm{p}}\right)\right]=-\frac{1}{2} \varphi_{\mathrm{n}}^{\dagger}\left(\sigma_{\mathrm{n}}\right)\left[3(\vec{\sigma} \cdot \vec{v}) \vec{v}-\vec{v}^{2} \vec{\sigma}\right] \varphi_{\mathrm{p}}\left(\sigma_{\mathrm{p}}\right),
\end{aligned}
$$

where $\vec{v}=\vec{k} / \sqrt{\vec{k}^{2}+M_{\mathrm{N}}^{2}}=\sqrt{1-4 M_{\mathrm{N}}^{2} / s}$ and $\vec{k}$ are a relative velocity and a 3 -momentum of the neutron-proton pair, $\varphi_{\mathrm{n}}\left(\sigma_{\mathrm{n}}\right)$ and $\varphi_{\mathrm{p}}\left(\sigma_{\mathrm{p}}\right)$ are spinorial wave functions of the neutron and the proton, respectively. It is obvious that the densities Eq.(2.11) describe the 
neutron-proton pair in the $\mathrm{S}$ - and $\mathrm{D}$-wave states with a total spin $S=1$ and a total momentum $J=1$.

The neutron-proton densities Eq.2.11) are normalized by

$$
\begin{aligned}
& \frac{1}{3} \sum_{\sigma_{\mathrm{n}}= \pm 1 / 2} \sum_{\sigma_{\mathrm{p}}= \pm 1 / 2} \vec{\Psi}^{\dagger}\left({ }^{3} \mathrm{~S}_{1} ; \sigma_{\mathrm{n}}, \sigma_{\mathrm{p}}\right) \cdot \vec{\Psi}\left({ }^{3} \mathrm{~S}_{1} ; \sigma_{\mathrm{n}}, \sigma_{\mathrm{p}}\right)=1 \\
& \frac{1}{3} \sum_{\sigma_{\mathrm{n}}= \pm 1 / 2} \sum_{\sigma_{\mathrm{p}}= \pm 1 / 2} \vec{\Psi}^{\dagger}\left({ }^{3} \mathrm{D}_{1} ; \sigma_{\mathrm{n}}, \sigma_{\mathrm{p}}\right) \cdot \vec{\Psi}\left({ }^{3} \mathrm{D}_{1} ; \sigma_{\mathrm{n}}, \sigma_{\mathrm{p}}\right)=v^{4}=\left(1-\frac{4 M_{\mathrm{N}}^{2}}{s}\right)^{2} .
\end{aligned}
$$

The decomposition of the neutron-proton densities in the amplitude Eq.(2.5) into the densities with a certain orbital momentum we would carry out at leading order in the large $N_{C}$ expansion [1-3]. This would allow to consider the neutron and the proton as free particles obeying free equations of motion

$$
\begin{aligned}
\bar{u}^{c}\left(k_{\mathrm{n}}, \sigma_{\mathrm{n}}\right)\left(\hat{k}_{\mathrm{n}}+M_{\mathrm{N}}\right) & =0, \\
\left(\hat{k}_{\mathrm{p}}-M_{\mathrm{N}}\right) u\left(k_{\mathrm{p}}, \sigma_{\mathrm{p}}\right) & =0 .
\end{aligned}
$$

In order to express the neutron-proton densities in the amplitude Eq.(2.5) in terms of the projection operators Eq.(2.7), first, we have to exclude the term containing $\sigma_{\mu \nu}$. This can be carried out by using Gordon's identity

$$
\left[\bar{u}^{c}\left(k_{\mathrm{n}}, \sigma_{\mathrm{n}}\right) \sigma_{\mu \nu} u\left(k_{\mathrm{p}}, \sigma_{\mathrm{p}}\right)\right] \frac{\left(k_{\mathrm{n}}+k_{\mathrm{p}}\right)^{\mu}}{2 M_{\mathrm{N}}}=-\left[\bar{u}^{c}\left(k_{\mathrm{n}}, \sigma_{\mathrm{n}}\right) \gamma_{\nu} u\left(k_{\mathrm{p}}, \sigma_{\mathrm{p}}\right)\right]+\frac{k_{\nu}}{M_{\mathrm{N}}}\left[\bar{u}^{c}\left(k_{\mathrm{n}}, \sigma_{\mathrm{n}}\right) u\left(k_{\mathrm{p}}, \sigma_{\mathrm{p}}\right)\right] .
$$

Substituting Eq.(2.14) in Eq.(2.5) we get

$$
\begin{aligned}
& M\left(\mathrm{n}\left(\mathrm{k}_{\mathrm{n}}, \sigma_{\mathrm{n}}\right)+\mathrm{p}\left(\mathrm{k}_{\mathrm{p}}, \sigma_{\mathrm{p}}\right) \rightarrow \mathrm{D}\left(k_{\mathrm{D}}, \lambda_{\mathrm{D}}\right)\right)=2 i\left(g_{\mathrm{V}}+2 g_{\mathrm{T}}\right) e^{* \nu}\left(k_{\mathrm{D}}, \lambda_{\mathrm{D}}\right) \\
& \times\left\{\left[\bar{u}^{c}\left(k_{\mathrm{n}}, \sigma_{\mathrm{n}}\right) \gamma_{\nu} u\left(k_{\mathrm{p}}, \sigma_{\mathrm{p}}\right)\right]-\frac{2 g_{\mathrm{T}}}{g_{\mathrm{V}}+2 g_{\mathrm{T}}} \frac{k_{\nu}}{M_{\mathrm{N}}}\left[\bar{u}^{c}\left(k_{\mathrm{n}}, \sigma_{\mathrm{n}}\right) u\left(k_{\mathrm{p}}, \sigma_{\mathrm{p}}\right)\right]\right\} .
\end{aligned}
$$

In terms of $\mathcal{S}_{\nu}$ and $\mathcal{D}_{\nu}$ vectors $\gamma_{\nu}^{\perp}$ and $k_{\nu}$ are determined by

$$
\begin{aligned}
\gamma_{\nu}^{\perp} & =\frac{2 \sqrt{2}}{3}\left(M_{\mathrm{N}}+\sqrt{s}\right) \mathcal{S}_{\nu}-\frac{2}{3} \frac{s}{2 M_{\mathrm{N}}+\sqrt{s}} \mathcal{D}_{\nu} \\
k_{\nu} & =\frac{1}{3 \sqrt{2}}\left(4 M_{\mathrm{N}}^{2}-s\right) \mathcal{S}_{\nu}-\frac{1}{3} s \mathcal{D}_{\nu} .
\end{aligned}
$$

Substituting Eq.(2.16) in Eq.(2.15) and taking into account that $\left[\bar{u}^{c}\left(k_{\mathrm{n}}, \sigma_{\mathrm{n}}\right) \hat{P} u\left(k_{\mathrm{p}}, \sigma_{\mathrm{p}}\right)\right]=0$ we obtain

$$
\begin{aligned}
& M\left(\mathrm{n}\left(\mathrm{k}_{\mathrm{n}}, \sigma_{\mathrm{n}}\right)+\mathrm{p}\left(\mathrm{k}_{\mathrm{p}}, \sigma_{\mathrm{p}}\right) \rightarrow \mathrm{D}\left(k_{\mathrm{D}}, \lambda_{\mathrm{D}}\right)\right)=4 \sqrt{2} i\left(g_{\mathrm{V}}+2 g_{\mathrm{T}}\right) M_{\mathrm{N}} e^{* \nu}\left(k_{\mathrm{D}}, \lambda_{\mathrm{D}}\right) \\
& \times\left\{\left[\bar{u}^{c}\left(k_{\mathrm{n}}, \sigma_{\mathrm{n}}\right) \mathcal{S}_{\nu} u\left(k_{\mathrm{p}}, \sigma_{\mathrm{p}}\right)\right]+\eta_{\mathrm{d}}\left[\bar{u}^{c}\left(k_{\mathrm{n}}, \sigma_{\mathrm{n}}\right) \mathcal{D}_{\nu} u\left(k_{\mathrm{p}}, \sigma_{\mathrm{p}}\right)\right]\right\}= \\
& =4 \sqrt{2} i\left(g_{\mathrm{V}}+2 g_{\mathrm{T}}\right) M_{\mathrm{N}} e^{* \nu}\left(k_{\mathrm{D}}, \lambda_{\mathrm{D}}\right)\left[\Psi_{\nu}\left({ }^{3} \mathrm{~S}_{1} ; \sigma_{\mathrm{n}}, \sigma_{\mathrm{p}}\right)+\eta_{\mathrm{d}} \Psi_{\nu}\left({ }^{3} \mathrm{D}_{1} ; \sigma_{\mathrm{n}}, \sigma_{\mathrm{p}}\right)\right],
\end{aligned}
$$

where $\eta_{\mathrm{d}}$ describes the fraction of the $\mathrm{D}$-wave state in the wave function of the deuteron. It is equal to

$$
\eta_{\mathrm{d}}=\frac{1}{3 \sqrt{2}} \frac{2 g_{\mathrm{T}}-g_{\mathrm{V}}}{2 g_{\mathrm{T}}+g_{\mathrm{V}}} .
$$


For the derivation of Eqs.(2.17) and (2.18) we have set $s=M_{\mathrm{D}}^{2}$ and neglected the contribution of the binding energy of the deuteron in comparison with a nucleon mass $M_{\mathrm{N}}$. This means that $4 M_{\mathrm{N}}^{2}-s=0$ when compared with $M_{\mathrm{N}}^{2}$.

Using the relation Eq.(2.2) the parameter $\eta_{\mathrm{d}}$ takes the value

$$
\eta_{\mathrm{d}}=\frac{1}{3 \sqrt{2}} \frac{\sqrt{3}-\sqrt{2}}{\sqrt{3}+\sqrt{2}}=0.0238 .
$$

This agrees well with the value $\eta_{\mathrm{d}}=0.0256 \pm 0.0004$ that was used in low-energy nuclear phenomenology for the description of the realistic wave function of the deuteron within the potential model approach $[9,10]$.

\section{Conclusion}

We have shown that the NNJL model describes well in agreement with low-energy nuclear phenomenology [10] such a fine structure of the deuteron as a contribution of the $\mathrm{D}$-wave state. The calculation of the fraction of the $\mathrm{D}$-wave state to the wave function of the deuteron we have carried out at leading order in the large $N_{C}$ expansion [1]. This has allowed to treat the neutron and the proton as free particles on-mass shell [2] obeying free equations of motion. To the decomposition of the amplitude of the transition $n$ $+\mathrm{p} \rightarrow \mathrm{D}$ into the neutron-proton quantum field configurations having certain orbital momenta and corresponding to the $\mathrm{S}$ - and $\mathrm{D}$-wave states, respectively, we have applied a relativistically covariant partial-wave analysis invented by Anisovich et al. [8] for the description of nucleon-nucleon scattering with nucleon-nucleon pairs coupled in the states with certain orbital momenta.

The theoretical value of the $\mathrm{D}$-wave state fraction in the wave function of the deuteron $\eta_{\mathrm{d}}=0.0238$ calculated in the NNJL model agrees well with low-energy nuclear phenomenology giving $\eta_{\mathrm{d}}=0.0256 \pm 0.0004$ [10]. The former was quoted by Kamionkowski and Bahcall [9] for the parameterization of the realistic wave function of the deuteron in connection with the calculation of the astrophysical factor $S_{\mathrm{pp}}(0)$ for the solar proton burning $\mathrm{p}+\mathrm{p} \rightarrow \mathrm{D}+\mathrm{e}^{+}+\nu_{\mathrm{e}}$. The calculation of the contribution of the $\mathrm{D}$-wave state fraction of the wave function of the deuteron in agreement with low-energy nuclear phenomenology testifies that the NNJL model describes to full extent low-energy tensor nuclear forces playing an important role in low-energy nuclear physics on the whole and for the existence of the deuteron, in particular [11].

\section{Acknowledgement}

One of the authors (V. Ivanova) is grateful to her supervisor Prof. A. A. Choban for discussions. 


\section{References}

[1] A. N. Ivanov, H. Oberhummer, N. I. Troitskaya, and M. Faber, Eur. Phys. J. A 7, 519 (2000).

[2] A. N. Ivanov, H. Oberhummer, N. I. Troitskaya, and M. Faber, Eur. Phys. J. A 8, 129 (2000).

[3] A. N. Ivanov, H. Oberhummer, N. I. Troitskaya, and M. Faber, Eur. Phys. J. A 8, 233 (2000).

[4] R. Dymarz and F. C. Khanna, Nucl. Phys. A 516, 549 (1990).

[5] QUARKS AND NUCLEI, ed. W. Weise, World Scientific, Singapore, 1989.

[6] MESONS IN NUCLEI, ed. M. Rho and D. H. Wilkinson, Noth-Holland, Amsterdam, 1979.

[7] D. Allasia et al., Phys. Lett. B 174, 450 (1986).

[8] V.V. Anisovich, M.N. Kobrinsky, D.I. Melikhov, A.V. Sarantsev, Nucl. Phys. A 544, 747 (1992); V. V. Anisovich, D. I. Melikhov, B. Ch. Metch, and H. R. Petry, Nucl. Phys. A 563, 549 (1993).

[9] M. Kamionkowski and J. Bahcall, ApJ. 420, 884 (1994).

[10] J. R. Bergervoet et al., Phys. Rev. C 38, 15 (1988).

[11] J. M. Blatt and V. F. Weisskopf, in THEORETICAL NUCLEAR PHYSICS, John Wiley \& Sons, New York Chapman \& Hall Ltd, London, 1952. 